Rev Biomed 2002; 13:110-114.

\title{
Compartamiento de la insulina plasmática con el ejercicio aeróbico de baja intensidad en pacientes con factores de riesgo cardiovascular.
}

\section{Comunicación Breve}

Pedro Fuentes-González, Felipe G. Cantón-Cuevas, William Vargas-Cano.

Departamento de Medicina del Deporte, Facultad de Medicina, Universidad Autónoma de Yucatán, Mérida, Yucatán, México.

\section{RESUMEN.}

Introducción. Se realizó un estudio experimental, longitudinal de ensayo clínico en 10 pacientes de ambos sexos, con edades entre 25 y 65 años, con más de dos factores de riesgo cardiovascular, el objetivo del estudio fue demostrar el efecto del ejercicio aeróbico de baja intensidad, sobre las concentraciones de insulina plasmática.

Material y método. El grupo fue sometido a un programa de ejercicio aeróbico de baja intensidad en banda sinfín de 3 meses de duración en sesiones de 30 minutos por día, cinco días a la semana, con medición mensual de insulina plasmática.

Resultados. Se observó disminución de la insulina plasmática en el $70 \%$ de los pacientes, aun que la respuesta no fue uniforme, observándose mejores resultados en los sujetos que iniciaron el estudio con hiperinsulinemia.

(Rev Biomed 2002; 13:110-114)
Palabras clave: Ejercicio aeróbico, insulina plasmática, riesgo cardiovascular.

\section{SUMMARY.}

Plasmatic insulin behavior with low intensity aerobic excercise in patients with cardiovascular risk factors.

Introduction. An experimental, longitudinal of clinical assay was carried out on ten patients with more than two cardiovascular risk factors of both genders aged between 25 and 65 . The aint of this study was to demostrate the effect of low intensity aerobic excercise on plasmatic insulin levels.

Material and method. The group was submited to a low intensity aerobic excercise program with a schedule of 30 minutes a day, five days a week for two months.

Results. A decrease in the plasmatic insulin levels was observed in $70 \%$ of the patients, and although

Solicitud de sobretiros: M.C. William A. Vargas-Cano, Departamento de Medicina del Deporte, Facultad de Medicina, Universidad Autónoma de Yucatán, Calle 84-A x 59 y 59-A, Mérida, Yucatán, México. Tel. (999) 923-99-57

Recibido el 27/Sep./2000. Aceptado para publicación el 21/Junio/2001. 
P Fuentes-González, FG Cantón-Cuevas, W Vargas-Cano.

the response wasn't the same in all patients, the best results were observed in the patients who started the study with hyperinsulinemia.

\section{(Rev Biomed 2002; 13:110-114)}

Key words: Aerobic excercise, plasmatic insulin, cardiovascular risk factors.

\section{INTRODUCCIÓN.}

La hipertensión arterial esencial, la diabetes mellitus tipo II, la obesidad, la hipertrigliceridemia, la disminución del factor cardioprotector (HDL) y la disminución de la fibrinolisis son entidades interrelacionadas y conocidas como el sexteto de la muerte, Síndrome X, síndrome de resistencia a la insulina o síndrome metabólico y tienen como elemento común la resistencia periférica a la insulina (1). Por lo que es importante identificar esta resistencia a la insulina, puesto que de ello dependerá la influencia del tratamiento como el ejercicio, principalmente del tipo aeróbico (2)

Estas entidades clínicas son tratadas con fármacos, los cuales pueden producir reacciones adversas, que en ocasiones son graves en el hígado (3). Autores como Kiovisto, Rogers, Lehman (46) y otros mencionan los efectos beneficiosos del ejercicio en el metabolismo de la glucosa, prevención de la diabetes y en la resistencia a la insulina.

Aunque no se ha demostrado si la hiperinsulinemia por sí misma es un factor causal de la hipertensión arterial, existen estudios que indican que estas dos entidades se interrelacionan, con un aumento del riesgo de enfermedad isquémica del corazón. De ahí la importancia de disminuir las concentraciones plasmáticas de la hormona, previniendo la hiperinsulinemia en pacientes con factores de riesgo cardiovascular. Esto es de mayor trascendencia si consideramos que según reporte de Cuauhtémoc Vázquez, el $20 \%$ de la población nacional es hipertensa (7)

Se ha demostrado que el ejercicio aeróbico dosificado y bien controlado, produce cambios metabólicos como incremento del efecto hipoglucemiante de la insulina, con disminución de su secreción e incremento de las hormonas antirreguladoras (catecolaminas, glucagón, hormona del crecimiento y cortisol) (8). Esta disminución de la concentración plasmática de la insulina está en relación inversa a la potencia del ejercicio, cuando este es submáximo, por lo que este ejercicio es de utilidad en el tratamiento integral de los diabéticos $(9,10)$.

El objetivo del presente trabajo, fue determinar si el ejercicio aeróbico leve de baja intensidad, es capaz de producir cambios favorables en la concentración de insulina plasmática en pacientes con algún otro factor de riesgo de los descritos como parte del síndrome $\mathrm{X}$, al igual que lo produce el ejercicio aeróbico moderado e intenso.

\section{MATERIAL Y MÉTODO.}

Se realizó un estudio prospectivo, longitudinal, de ensayo clínico en 10 pacientes, 5 del sexo masculino y 5 del sexo femenino, entre 25 y 65 años de edad, con una media de 44.2 años. Cinco presentaron hiperinsulinemia y obesidad; en este grupo la edad media fue de 41.2 años; uno presentó además glicemia en ayuno de $167 \mathrm{mg} / \mathrm{dL}$ e hipercolesterolemia, dos hipercolesterolemia y dos hipertensión e hipercolesterolemia. Cinco pacientes, con un promedio de edad de 47.2 años, tuvieron valores normales de insulina plasmática, todos con obesidad, tres presentaron además hipertensión e hipercolesterolemia y uno sólo hipercolesterolemia. Ninguno habían realizado ejercicio físico durante los seis meses previos al estudio. Se procedió a la evaluación médica del grupo, que incluyó historia clínica, determinación de insulina medida en suero con Kit comercial Coat -a-count Insulin ( ${ }^{125} \mathrm{I}$ radioimmunoassay) tomándose como valor normal de 3 a $35 \mu \mathrm{UI} / \mathrm{mL}$. Se realizó prueba de esfuerzo diagnóstica en banda sinfín con protocolo de Bruce, para lo cual se solicitó estudios de laboratorio clínico: urea,

\section{Revista Biomédica}


Comportamiento de la insulina plasmática con el ejercicio.

creatinina, citometría hemática, colesterol total, fracciones HDL y LDL y triglicéridos con el fin de cumplir con los criterios establecidos por el laboratorio de ergometría del Departamento de Medicina del deporte, para la realización de una prueba de esfuerzo diagnóstica.

El laboratorio clínico y la prueba de esfuerzo se realizó en forma mensual durante el tiempo que duró el estudio.

La toma de muestras para análisis de laboratorio, se realizó por la mañana con 14 horas de ayuno, sin haber realizado actividad física 24 horas antes.

La prueba de esfuerzo con protocolo de Bruce, se realizó en ayuno de 3 a 6 horas, sin haber realizado esfuerzo físico las 24 horas previas a la prueba.

El grupo de estudio fue sometido a un programa de ejercicio físico aeróbico de intensidad leve, en banda sinfín, de tres meses de duración con sesiones de 30 minutos, una vez al día, 3 a 5 días a la semana, con monitoreo cardíaco y medición de la tensión arterial cada 6 minutos durante las sesiones. Durante todo el tiempo que duró el programa de ejercicio, se cuidó que los pacientes no se realizaran cambios en su dieta ni en sus otros patrones de vida.

Características del programa de ejercicio.

Parámetros: Para determinar la intensidad del ejercicio, se utilizó la frecuencia cardíaca máxima estimada por edad del paciente.

Duración: 5 meses en sesiones de 30 minutos.

Frecuencia: Una sesión por día, 3 a 5 días a la semana.

Intensidad: $55 \%$ de la frecuencia cardíaca máxima, determinada por edad del paciente.

Progresión: La progresión únicamente se realizó para mantener el $55 \%$ de la frecuencia cardiaca máxima del paciente determinada por edad, durante todo el programa.

\section{RESULTADOS.}

Todos los pacientes que iniciaron el programa con hiperinsulinemia, redujeron su valores a cifras normales al final del estudio (cuadro 1).

Sin embargo, el comportamiento de la insulina no fue uniforme y por ser la muestra muy pequeña, no se puede sugerir si existe relación entre el número y tipo de factores de riesgo y el comportamiento de la insulina (cuadro 1).

En cuanto al grupo normoinsulínico, al final del estudio dos disminuyeron sus valores plasmáticos de insulina, uno no presentó cambios y dos presentaron incremento; éstos presentaron como factores de riesgo agregado obesidad, hipertensión e hipercolesterolemia (figura 1).

En este grupo al igual que en el de hiperinsulinémicos, durante el programa de ejercicio se observó variaciones en la insulina, pero manteniéndose siempre los niveles dentro del rango considerado como normal (cuadro 2).

En cuanto a los otros parámetros de laboratorio controlados, en el grupo de los hiperinsulinémicos, el HDL, aumentó en 3 pacientes y los triglicéridos disminuyeron en los 5

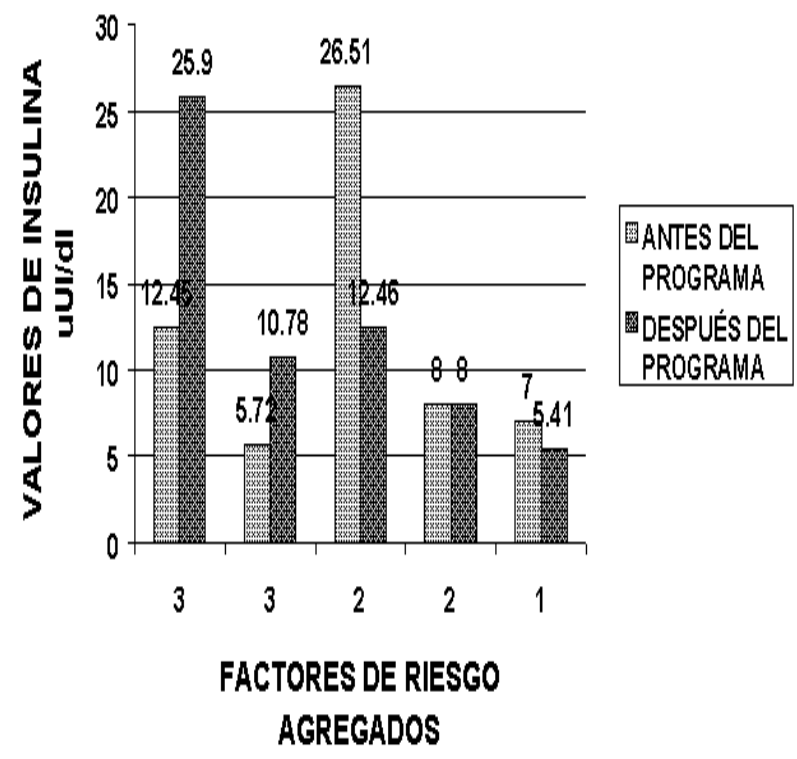

Figura 1.- Comportamiento de la insulina con un programa de ejercicio aeróbico, en pacientes normoinsulínicos.

Vol. 13/No. 2/Abril-Junio, 2002 
P Fuentes-González, FG Cantón-Cuevas, W Vargas-Cano.

Cuadro 1

Comportamiento de la insulina plasmática en pacientes hiperinsulinémicos, con un programa de ejercicio aeróbico de tres meses de duración.

\begin{tabular}{|c|c|c|c|c|c|c|c|}
\hline \multirow[t]{2}{*}{ Paciente } & \multirow[t]{2}{*}{ Edad } & \multirow[t]{2}{*}{ Género } & \multirow{2}{*}{$\begin{array}{c}\text { Factores } \\
\text { de } \\
\text { Riesgo }\end{array}$} & \multicolumn{4}{|c|}{$\begin{array}{l}\text { Valor de Insulina en Sangre } \mu \mathrm{UI} / \mathrm{mL} \\
\text { Meses de Ejercicio }\end{array}$} \\
\hline & & & & Basal & 1 & 2 & 3 \\
\hline 01 & 27 & $\mathrm{~F}$ & 2 & 40.00 & 6.82 & 10.91 & 7.22 \\
\hline 02 & 35 & M & 3 & 37.71 & 9.01 & 77.39 & 7.21 \\
\hline 03 & 40 & M & 3 & 66.09 & 108.07 & 31.02 & 32.29 \\
\hline 04 & 43 & M & 3 & 37.62 & 59.10 & 14.37 & 30.48 \\
\hline 05 & 61 & $\mathrm{~F}$ & 2 & 58.50 & 14.29 & 34.03 & 15.00 \\
\hline 06 & 32 & F & 2 & 8.00 & 9.36 & 8.71 & 8.00 \\
\hline 07 & 38 & M & 3 & 5.72 & 0.69 & 27.55 & 10.78 \\
\hline 08 & 51 & M & 3 & 12.54 & 26.10 & 27.68 & 25.90 \\
\hline 09 & 55 & $\mathrm{~F}$ & 2 & 26.51 & 38.29 & 13.81 & 12.46 \\
\hline 10 & 60 & F & 1 & 7.00 & 7.74 & 5.86 & 5.41 \\
\hline
\end{tabular}

pacientes (cuadro 2). En el grupo de los pacientes normoinsulinémicos, se observó incremento del HDL en 3 pacientes, y disminución de los triglicéridos en 4 pacientes (cuadro 3).

\section{DISCUSIÓN.}

El ejercicio aeróbico de baja intensidad, produjo disminución de los niveles plasmáticos de insulina en los 5 pacientes que iniciaron el programa con hiperinsulinemia y solamente en dos de los 5 que iniciaron sin hiperinsulinemia. Sin embargo el comportamiento no fue uniforme en ningún grupo, pero por el tamaño de la muestra no se puede afirmar si las variaciones tienen alguna relación con el valor plasmático de insulina previo al estudio.
Sin embargo todos los que iniciaron el programa con valores de insulina por arriba del parámetro normal, al final del estudio presentaron valores normales, con disminución que fluctuó entre el 18.9 y el $74.3 \%$ del valor inicial. Por otro lado, los pacientes que iniciaron el programa sin hiperinsulinemia, presentaron fluctuaciones de la hormona durante el programa de ejercicio, pero manteniéndose siempre dentro de los valores considerados como normales.

Con relación al HDL y los triglicéridos, la respuesta fue más uniforme: en ambos grupos el HDL se incremento en 3 pacientes. Los triglicéridos disminuyeron en 4 pacientes normoinsulinémicos y en los 5 hiperinsulinémicos. Los resultados sugieren que el ejercicio aeróbico

\section{Cuadro 2}

Comportamiento del HDL y los triglicéridos con un programa de ejercicio aeróbico de tres meses de duración, en pacientes hiperinsulinémicos

\begin{tabular}{llllllllll}
\hline & & \multicolumn{2}{c}{ HDL } & & \multicolumn{4}{c}{ TRIGLICÉRIDOS } \\
& BASAL & \multicolumn{2}{c}{ MESES DE EJERCICIO } & BASAL & \multicolumn{2}{c}{ MESES DE EJERCICIO } \\
& & 1 & 2 & 3 & & 1 & 2 & 3 \\
\hline 1 & 37 & 30.2 & 31.3 & 40.00 & 149 & 152 & 121 & 109 \\
2 & 39.3 & 35.4 & 34.7 & 34 & 439 & 445 & 299 & 287 \\
3 & 50.9 & 61.9 & 38 & 50 & 155 & 129 & 111 & 105 \\
4 & 42 & 40.5 & 44.8 & 41 & 319 & 369 & 380 & 239 \\
5 & 31 & 38.9 & 35.6 & 38 & 271 & 265 & 237 & 165 \\
\hline
\end{tabular}




\section{Comportamiento de la insulina plasmática con el ejercicio.}

Cuadro 3

Comportamiento del HDL y los triglicéridos con un programa de ejercicio aeróbico de tres meses de duración, en pacientes normoinsulinémicos.

\begin{tabular}{lllllllll}
\hline & & \multicolumn{3}{c}{ HDL } & & \multicolumn{4}{c}{ TRIGLICÉRIDOS } \\
& BASAL & \multicolumn{2}{c}{ MESES DE EJERCICIO } & BASAL & \multicolumn{2}{c}{ MESES DE EJERCICIO } \\
& & 1 & 2 & 3 & & 1 & 2 & 3 \\
\hline 6 & 47.5 & 31 & 7 & 21 & 190 & 520 & 560 & 640 \\
7 & 60.01 & 62 & 62 & 62 & 160 & 194 & 194 & 135 \\
8 & 54.9 & 65 & 53 & 51 & 282 & 193 & 223 & 226 \\
9 & 42.7 & 39.6 & 38.5 & 57 & 347 & 348 & 483 & 225 \\
10 & 38 & 61.6 & 32.5 & 47.4 & 277 & 213 & 178 & 148 \\
\hline
\end{tabular}

leve puede ser útil como parte de la terapéutica de los pacientes con factores de riesgo, ya que todos los que iniciaron con hiperinsulinemia normalizaron sus valores de insulina y los normoinsulinémicos mantuvieron sus valores dentro de los parámetros normales, durante todo el tiempo que duró el programa de ejercicio. Sin embargo el grupo de estudio fue muy pequeño para poder inferir los resultados a la población de riesgo, por lo que sería conveniente verificar este tipo de estudio con una muestra significativa y durante un lapso mayor.

\section{REFERENCIAS.}

1.- Fanghänel G. Enfoque multifactorial del Síndrome de Resistencia a la Insulina. Rev Endocr Nutr 1993;1:50.

2.- Brito-Zurita O. Influencia de la resistencia a la insulina en la hipertensión arterial esencial y la cardiopatía coronaria. Rev Endocr Nutr 1993;1:53.

3.- Nilsson P, Eriksson KF. New Drugs against insulin resistence. Lakartidningen 1998; 10:2832-4.

4.- Kiovisto VA, Yki-Jarvine, DeFronzo RA. Physical training and Insulin sensitivity. Diabetes Metab Rev 1986; 4: 445-81.

5.- Lehman R, Spinas GA. Role of physical activity in the therapy and prevention of type II diabetes mellitus. Ther Umsch 1996; 12: 925-33.

6.- Rogers MA. Acute effects of exercise on gluccose tolerance in non.insulun-dependent diabetes. Med Sci Sports Exerc 1989; 4: 362-8.
7.- Vasquez-Chávez C. Concentraciones de insulina en población sana e hiperinsulinemia en población mexicana hipertensa. Rev Endocr Nutr 1993;1:55.

8.- Zinman B, Viranic M. Diabetes y Ejercicio. Med Clin North Am 1985;1:162-3.

9.- Lehman R, Spinas GA. Role of physical activity in the therapy and prevention of tipe II diabetes mellitus. Ther Umsch 1996; 53:925-33.

10.- Trovati M, Carta Q, Cavalot F. Influence of physical training on blood glucose control, glucose tolerance, insulin cecretion, and insulin action in non-insulin-dependent diabetes. Diabetes Care 1984; 7:416-20. 